\title{
Efficacy and Safety of VB-1953 Topical Gel in Non-Responder Acne Patients with Clindamycin-Resistant Cutibacterium acnes
}

\author{
Rohit Batra ${ }^{1} \cdot$ Suresh Sadhasivam ${ }^{2} \cdot$ Swamini Saini $^{2} \cdot$ Swati Gupta ${ }^{2} \cdot$ Rahul Kumar Singh Bisen $^{2} \cdot$ Mau Sinha $^{2}$. \\ Shamik Ghosh ${ }^{2}$. Shilpi Jain ${ }^{2}$
}

Published online: 28 March 2020

(c) The Author(s) 2020

\begin{abstract}
Background and Objectives The emergence of resistant strains of Cutibacterium acnes can limit the efficacy of currently approved antibiotics for acne. VB-1953 is a next-generation antibiotic that exerts a bactericidal effect on resistant C. acnes. In this study, we investigated the safety, tolerability, and efficacy of VB-1953 topical gel in patients with moderate to severe acne having clindamycin-resistant $C$. acnes.

Methods An investigator-initiated, open label, single-arm clinical study was conducted in patients with moderate to severe facial acne vulgaris showing poor or no response to previous clindamycin treatment. Nineteen subjects were enrolled in the study based on laboratory screening for the presence of clindamycin-resistant $C$. acnes in acne swab samples collected from patients. VB-1953 2\% gel was applied on the entire face twice daily over 12 weeks. The primary efficacy endpoints were absolute changes in inflammatory and noninflammatory lesion counts from baseline at week 12, while the secondary efficacy endpoint was the proportion of subjects achieving Investigator Global Assessment success (score of 0 or 1 ) or a grade 2 or higher improvement from baseline at week 12. The presence and severity of local skin reactions (erythema, edema, scaling/ dryness, burning/stinging, pruritus) were evaluated for safety. Additionally, the detection and quantification of drug-resistant C. acnes strains were performed in the laboratory using acne swab samples collected from patients.

Results The occurrence of treatment-emergent adverse events or changes in vital signs, physical examinations, and urinalysis for any of the patients during the course of the entire study were clinically insignificant. Topical application of 2\% VB-1953 topical gel resulted in a significant reduction of mean absolute inflammatory and noninflammatory lesion counts by $53.1 \%$ and 52.2\%, respectively ( $p<0.0001$ for both), with an Investigator Global Assessment success of $26.3 \%$ at week 12 compared with baseline. Resistant bacteria were reduced by $(94.3 \pm 1 \% ; p<0.05)$ within 12 weeks of treatment with VB-1953. Conclusion These results indicate that VB-1953 topical gel can be a safe and effective therapy for moderate to severe acne with underlying resistant $C$. acnes in subjects who had not responded to previous antibiotic treatments.
\end{abstract}

Electronic supplementary material The online version of this article (https://doi.org/10.1007/s40268-020-00299-z) contains supplementary material, which is available to authorized users.

Rohit Batra

drrohitbatra36@gmail.com

Shilpi Jain

article@vyometx.com

Dermaworld Skin and Hair Clinic, New Delhi, India

2 Vyome Therapeutics Limited, Delhi, India

\section{Key Points}

The efficacy and safety of the bactericidal antibiotic VB-1953 (2\%) topical gel was evaluated for acne treatment in an investigator-initiated, open-label, single-arm study in acne patients who did not respond to or showed poor response to previous clindamycin treatment and had clindamycin-resistant Cutibacterium acnes.

In the era of emerging antimicrobial resistance, the selection of VB-1953 for acne treatment can play a key role in improving clinical outcomes in acne patients and in public health. 


\section{Introduction}

Acne vulgaris is a chronic inflammatory skin disease that is predominant in late childhood and adolescence but can occur at any age and can persist well into adulthood [1-3]. The clinical features of acne include seborrhoea, inflammatory (pustules, papules, nodules or cysts) and noninflammatory lesions (open and closed comedones), as well as scarring in severe cases [3-5]. Although there are many facets of acne pathogenesis, one of the key etiological factors in its development is Gram-positive Cutibacterium acnes (C. acnes) [6]. The host response to $C$. acnes can result in the production of various proinflammatory cytokines, including tumour necrosis factor (TNF)- $\alpha$ and several interleukins (ILs) such as IL-1, IL-6, IL-8 and IL-12 [7-9]. C. acnes activates toll-like receptor (TLR)-2 for triggering host-mediated inflammation, which in turn modulates the nuclear factor-kappa B (NF- $\kappa \mathrm{B})$ pathway $[8,10]$. Furthermore, proteases secreted from $C$. acnes induce protease-activated receptor (PAR)-2, which leads to the release of IL-8 and matrix metalloproteinases (MMPs) from keratinocytes [11]. In macrophages, $C$. acnes-stimulated reactive oxygen species (ROS) can induce the expression of inducible nitric oxide synthase and cyclooxygenase-2/prostaglandin E2 (COX-2/PGE2) during acne infection [12]. Moreover, literature reports indicate that in human sebocytes, $C$. acnes stimulates the NLRP3 infammasome, which regulates the maturation and secretion of IL-1 $\beta$ via caspase- 1 activity [13]. Antibiotics are the first choice of treatment for mild-to-moderate, mixed, papular/pustular, and moderate nodular acne, and an alternative choice in severe, nodular/conglobate acne [14, 15]. The widespread use of oral and topical antibiotics such as macrolides, lincosamides and tetracyclines to treat acne, sometimes for extensive periods, has led to the worldwide emergence of $C$. acnes strains resistant to these antibiotics [16-18].

Multiple studies reporting the detection of resistant isolates of $C$. acnes were published in different parts of the world [19-21]. In the US, resistance to clindamycin has evolved to a staggering one in three patients carrying resistant strains of $C$. acnes [17]. Clindamycin-resistant $C$. acnes were classified into different classes based on the point mutations observed in the $23 \mathrm{~S}$ ribosomal RNA (rRNA) gene of C. acnes, i.e. A2058G (class I), A2059G (class IV) and G2057A (class III) [Escherichia coli equal numbering] [22, 23]. The mechanism of drug resistance in phenotype class II is mediated by the ermX gene present in the mobile genetic element, transposon TN5432 of Corynebacterial origin [24], while tetracycline resistance is mediated by point mutation $\mathrm{G} 1058 \mathrm{C}$ in $16 \mathrm{~S}$ rRNA of $C$. acnes [25].

The present treatment guidelines recommend antibiotics in combination with other antibacterials to achieve disease control by attaining maximal outcome in the management of inflammatory and noninflammatory acne, as well as to avoid resistance development [26, 27]; however, this regimen may only be partially effective in cases of patients harboring resistant strains [18]. This challenging situation demands the development of new antibiotics with potent bactericidal action against $C$. acnes and additionally exhibiting anti-inflammatory properties.

We developed a novel topical gel formulation of VB-1953 (2\%), a fourth-generation fluroquinolone, effective against $C$. acnes. Fluoroquinolones are known to interfere with the activities of enzymes, DNA gyrase and topoisomerase IV, essential for bacterial DNA replication [28]. A fluoroquinolone-based anti-acne drug, nadifloxacin, having bactericidal action against C. acnes, is approved in parts of Europe and Japan for acne treatment [29, 30]. One of our previous reports has shown potent in vitro activity of VB-1953 against clinical isolates of $C$. acnes with diverse resistance spectrum (mutations in either A2058G or A2059G [E. coli numbering] in bacterial $23 \mathrm{~S}$ rRNA) to clindamycin and erythromycin [18]. Recently, the VB-1953 formulation resulted in a significant reduction of inflammatory lesions after 12 weeks of application of the formulation in patients with moderate to severe acne in a proofof-concept randomised trial [31]. Additionally, the formulation was found to be safe and well tolerated in humans. Based on these observations, we rationalised that VB-1953 could emerge as a novel treatment for inflammatory acne in patients carrying clindamycin-resistant $C$. acnes and not responding to clindamycin treatment.

\section{Methods}

\subsection{Study Participants}

Healthy male or non-pregnant females aged between 18 and 45 years with a clinical diagnosis of acne vulgaris of moderate to severe grade (grade 3 or 4 as determined by the Investigator's Global Assessment [IGA]) were included in this study. Subjects had at least 20 inflammatory lesions (papules, pustules and nodules/cysts) and at least 20 noninflammatory lesions (open/closed comedones) on the face. Subjects with more than two facial nodulocystic lesions, active nodulocystic acne, and any skin condition or disease interfering with evaluation were excluded. Furthermore, all subjects enrolled in the study had undergone topical clindamycin or clindamycin fixed-dose combination (FDC) treatment in the past month, did not or poorly responded to clindamycin treatment, and harbored clindamycin-resistant C. acnes strains. Moreover, subjects with serious clinical illness or chronic diseases, and subjects who had taken certain topical or systemic anti-acne treatments that, in the opinion of the investigator, may interfere with the study, were not included. The inclusion and exclusion criteria for subject 
recruitment are described in detail in the electronic supplementary Methods, Sect. 1.1.

\subsection{Study Design}

This was an open label, non-randomised, prospective clinical study evaluating the safety, tolerability and efficacy of VB-1953 in adult subjects with moderate to severe facial acne vulgaris who did not respond, or had low response, to clindamycin treatment. On Visit 1, an initial screening was performed, followed by collection of a bacterial swab from the patient's face, as mentioned in Sect. 2.5. Thereafter, microbiological testing was performed to check the presence of clindamycin-resistant strains in the samples collected. Of the 80 patients screened, samples from 19 subjects were positive for the above strains, which called for further follow-up visits. On Visit 2, the 19 subjects were enrolled into the study and were assessed for baseline IGA, lesion counts (inflammatory and noninflammatory) and local skin reactions (LSRs). Subjects were instructed to apply VB-1953 (2\%) gel on the entire face twice daily for 12 weeks. Visit 2 was then followed by three subsequent visits, i.e. Visits 3 , 4 and 5, corresponding to 4, 8 and 12 weeks after Visit 2, respectively. Furthermore, acne swab samples from enrolled patients were collected on Visit 3 (week 4) and Visit 5 (week 12) for microbiology testing.

The study was approved by the Independent Ethics Committee (Good Society of Ethical Research [GSER], Delhi, India) and was conducted as per Schedule Y (amended version, 2005) of the Central Drugs Standard Control Organization (CDSCO), Ministry of Health and Family Welfare, Government of India; 'Ethical Guidelines for Biomedical Research on Human Participants' (2006); Indian Council of Medical Research (ICMR)]; International Conference on Harmonization (ICH) E6-(R1) 'Guideline for Good Clinical Practice'; and Declaration of Helsinki (2013). Study-related procedures were initiated only after obtaining written informed consent from all the subjects.

\subsection{Study Treatment and Outcome Measurements}

All subjects applied VB-1953 topical gel (2\%) twice daily (in the morning and evening) for 12 weeks, and were encouraged to report any adverse incidences that happened during the study period. Safety was assessed by evaluation of local and systemic adverse events (AEs) and physical examination. The LSRs of erythema, edema, and scaling/dryness were estimated using a 5-point scale, and the LSRs of stinging/burning and pruritus/itching were estimated using a 4-point scale [32-34].
Efficacy was determined by acne lesion counts (inflammatory and noninflammatory lesions) and the IGA of overall acne severity (a 5-point static scale). Analysis as per the statistical analysis plan was conducted on the following parameters: (1) absolute change in inflammatory lesions from baseline to week 12; (2) absolute change in noninflammatory lesions from baseline to week 12; (3) proportion of subjects with IGA success.

The presence of clindamycin-resistant $C$. acnes in the acne swab samples collected from patients was checked and quantitated by microbiology and molecular biology testings.

\subsection{Study Analyses}

All statistical processing was performed using R software (The R Foundation for Statistical Computing, Vienna, Austria). Summary tables (descriptive statistics and/or frequency tables) were prepared for baseline, efficacy and safety variables. Continuous variables were described using descriptive statistics (mean, median, standard deviation, minimum and maximum), and the frequency counts and percentage of subjects within each category were provided for categorical data. $P$ values $<0.05$ were considered descriptive and no adjustments were made for multiple comparisons. Descriptive statistics were used to summarise compliance for the safety population. Measures of compliance included the duration of treatment (days) and the total number of applications. The efficacy endpoints, and the absolute change from baseline in inflammatory and noninflammatory lesion counts at week 12, were analysed using the paired $t$ test. The proportion of subjects with IGA success was analysed using the Cochran-Mantel-Haenszel (CMH) test for general association stratified by site.

AEs were coded using the Medical Dictionary for Regulatory Activities (MedDRA) coding dictionary and summarised according to relationship to the test article, and severity. Changes in physical examination, vital signs (temperature, blood pressure, pulse rate and respiration rate) were summarised using descriptive statistics. The analysis of safety was conducted on the safety population.

\subsection{Detection of Clindamycin-Resistant Cutibacterium acnes in Acne Lesions of Patients}

\subsubsection{Swab Collection}

Swab samples were collected from three places on the subject's face, following previously described detergent scrub/ swab methods with modifications [35]. To remove the aerobic bacteria and dirt on the surface, the volunteer's forehead and cheeks were cleaned by thoroughly wiping the area for $30 \mathrm{~s}$ with sterile gauze soaked in $0.1 \%$ Triton-X-100. By 
holding the sterile plastic ruler and marker, the sampling area $\left(16 \mathrm{~cm}^{2}[4 / 4 \mathrm{~cm}]\right)$ was marked in three places on the face (left cheek, right cheek and forehead). The swab sample was collected from all three places by rubbing the moistened sterile cotton swab for $30 \mathrm{~s}$. All three cotton swabs were transferred, one after the other, into a sterile falcon tube containing $2 \mathrm{~mL}$ of sterile fluid thioglycolate broth, and the bacterial content was released by squeezing the cotton swab tip against the falcon tube wall. Samples were brought to the microbiology laboratory as early as possible to analyse the presence of $C$. acnes/drug-resistant $C$. acnes by conventional microbiology and molecular biology assays.

\subsubsection{Microbiology Culture-Based Identification of Clindamycin-Resistant C. acnes}

Skin swab samples were serially diluted using sterile brain heart infusion (BHI) broth, and $100 \mu \mathrm{L}$ of sample was spread on BHI agar, BHI agar with $4 \mu \mathrm{g} / \mathrm{mL}$ furazolidone, BHI agar with $0.4 \mu \mathrm{g} / \mathrm{mL}$ clindamycin and BHI agar with $8 \mu \mathrm{g} / \mathrm{mL}$ clindamycin. Plates were incubated for 7-10 days under anaerobic conditions [36]. After incubation, depending on bacterial growth and the number of putative $C$. acnes counts (minute white colonies), 1-10 colonies were subcultured into fresh $\mathrm{BHI}$ agar plates and incubated for a further 2 days. After incubation, 1-5 $C$. acnes characteristic colonies from each plate were tested for further identification. Each suspected colony was subjected to genomic DNA extraction, and further polymerase chain reaction (PCR) amplification was performed for species-level confirmation, identification of A2058G (E. coli numbering) mutations in the 23S rRNA gene by restriction enzyme digestion, and presence of the ermX gene by PCR. The presence of $C$. acnes in the samples was confirmed by amplification of the $C$. acnes $23 \mathrm{~S}$ rRNA gene using species-specific primers. The colonies that successfully amplified and provided the expected band (646 bp) in agarose gel electrophoresis were confirmed as $C$. acnes (electronic supplementary Fig. 1a). The association of clindamycin-resistant $C$. acnes was determined by restriction enzyme digestion of $646 \mathrm{bp}$ PCR product with the MboII restriction enzyme [37]. It specifically recognises the point mutation A2058G ( $E$. coli numbering) responsible for clindamycin resistance and cleaves the $646 \mathrm{bp}$ product into two products $(450 \mathrm{bp}$ and $200 \mathrm{bp}$, respectively) (electronic supplementary Fig. 1b). Clindamycin resistance mediated by ermX was determined using erm $\mathrm{X}$ gene-specific primers (electronic supplementary Fig. 1c).

\subsubsection{Direct Quantification of Clindamycin-Resistant C. acnes Using the Real Time-Polymerase Chain Reaction Method}

The real time-PCR (RT-PCR) method was developed to quantify the number of clindamycin-resistant $C$. acnes present in acne samples. Genomic DNA was extracted from the samples directly and quantitative PCR (qPCR) amplification was performed using specific primers and fluorescencelabelled (FAM) minor groove binder (MGB) [38] probes to specifically detect A2058G mutations in the 23S rRNA gene of $C$. acnes (electronic supplementary Fig. 2). Different cell densities of the clindamycin-resistant $C$. acnes strain (CCARM 9010) were used as standard for quantification of the bacterial load. Approximately $600 \mu \mathrm{L}$ of swab sample and CCARM 9010 C. acnes strain (in different dilutions from $10^{1}$ to $10^{8} \mathrm{CFU} / \mathrm{mL}$ ) were subjected to total bacterial genomic DNA extraction using a QIAGEN kit (Germantown, MD, USA) as per the manufacturer's instructions. Moreover, the genomic DNA was subjected to qPCR quantification using a fluorescence-labelled probe (FAM) and primers to enumerate the number of clindamycin-resistant C. acnes present in the sample [38, 39].

\section{Results}

\subsection{Study Population}

Eighty subjects were screened for all study inclusion parameters and 19 subjects were finally enrolled in the trial. The study flowchart is shown in Fig. 1. All subjects completed the trial and were evaluated for safety and efficacy assessment. The enrolled subjects included both males (63.2\%) and females (36.8\%) in the 18-29 years age group, with a mean age of 21.8 years. Demographics of the subject population are shown in Table 1.

\subsection{Safety Assessments}

No occurrence of treatment-emergent AEs (TEAEs; local or systemic) or changes in vital signs, physical examinations and urinalysis were reported for any patients during the course of the entire study. A negligible number of LSRs (erythema, edema, scaling, stinging/burning, and pruritus/ itching) were reported in the study subjects, with all LSRs being predominantly minimal in severity on all visits, from Visit 2 to Visit 5 (Table 2). One instance of a moderate LSR was observed in a case of stinging/burning and scaling on Visit 2 but was not observed in subsequent visits. 
Fig. 1 Subject flow diagram for investigator-initiated trial. Study period: 12 weeks; 6 November 2017 (date of first subject enrolled), 30 August 2018 (date of last subject completed)

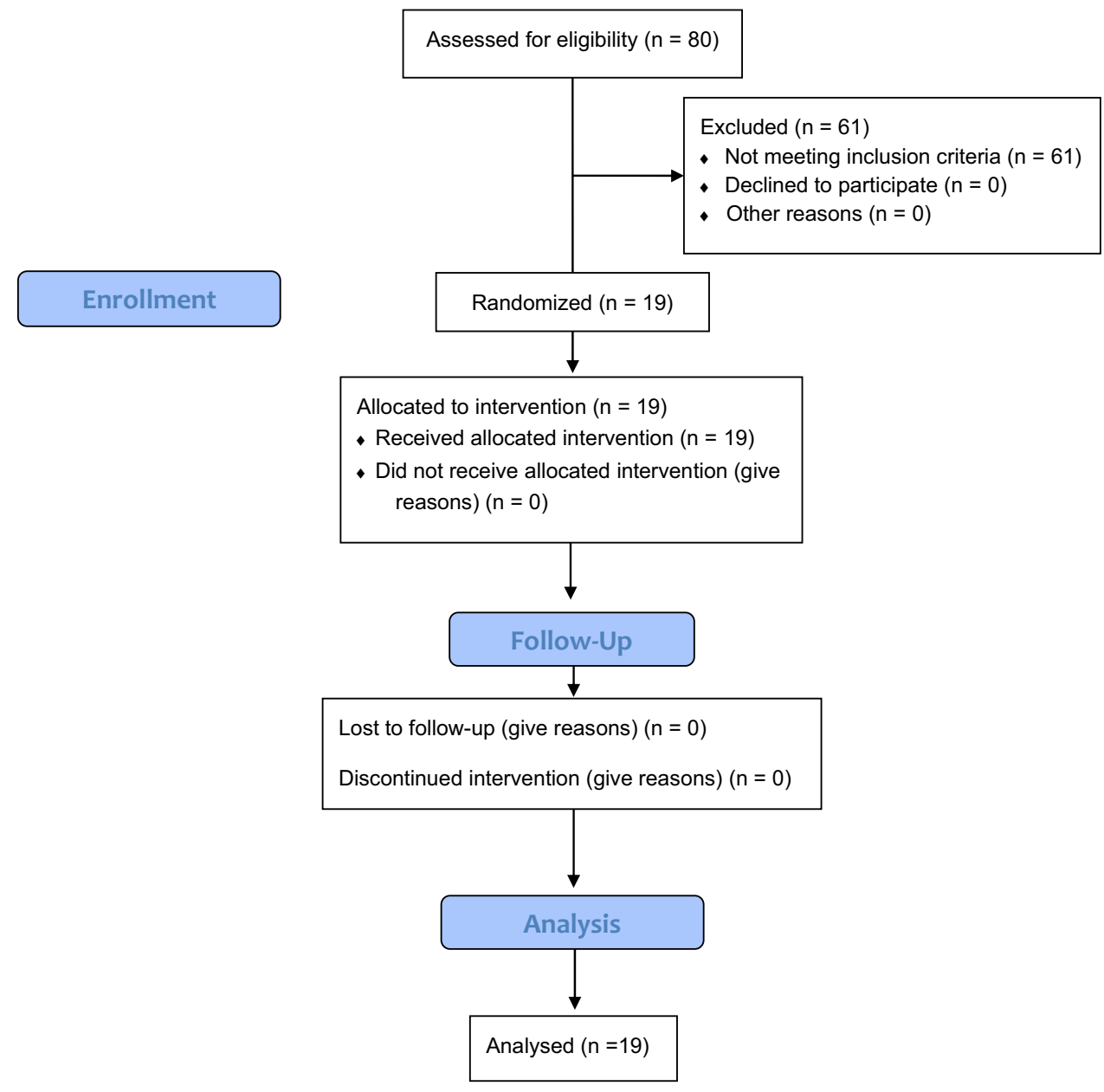

Table 1 Demographics of the safety and efficacy population

\begin{tabular}{ll}
\hline Characteristics & $\begin{array}{l}\text { Safety and } \\
\text { efficacy popu- } \\
\text { lation }\end{array}$ \\
\hline $\begin{array}{l}\text { Total number } \\
\text { Age, years }\end{array}$ & 19 \\
Mean (SD) & $21.8(3.2)$ \\
Median & 21.0 \\
Range & $18-29$ \\
Sex & \\
Number of females $(\%)$ & $7(36.8)$ \\
Number of males $(\%)$ & $12(63.2)$ \\
\hline
\end{tabular}

$S D$ standard deviation

\subsection{Efficacy Assessments}

There was marked improvement in both inflammatory and noninflammatory lesions after 12 weeks of VB-1953 (2\%) topical application, from baseline (Fig. 2a, b). The mean inflammatory lesion count decreased from $34.4 \pm 6.4$ at baseline to $16.7 \pm 9.0$ at week 12 , with a mean reduction from baseline of $53.1 \%(p<0.0001)$ (Fig. 2c). Similarly, for noninflammatory lesion count, the mean absolute value reduced from $39.4 \pm 7.8$ at baseline to $19.5 \pm 10.0$ at week 12 , with a mean reduction from baseline of $52.2 \%(p<0.0001)$ (Fig. 2c).

IGA success at week 12 after topical application of VB-1953 gel (2\%) was $26.3 \%$, where IGA success was defined as a score of 'clear' or 'almost clear' (IGA score of 0 or 1) and a grade 2 or higher IGA improvement from baseline. The number of subjects enrolled in the study who had moderate (IGA score of 3) or severe (IGA score of 4) acne at baseline was 18 and 1, respectively. At week 12, five subjects had an IGA score of 1 (almost clear), nine subjects had an IGA score of 2 (mild), and five subjects had an IGA score of 3 (moderate) (Fig. 2d). Overall, 31.6\% and 47.1\% of subjects exhibited a 2-point and 1-point improvement in IGA score, respectively, while $21.1 \%$ of subjects exhibited no change in IGA score compared with baseline at week 12 . 
Table 2 Summary of local skin reactions after application of VB-1953: safety of VB-1953

\begin{tabular}{|c|c|c|c|c|c|}
\hline \multirow{2}{*}{$\begin{array}{l}\text { LSR param- } \\
\text { eters }\end{array}$} & \multicolumn{2}{|l|}{ Visit 2} & \multirow{2}{*}{$\begin{array}{l}\text { Visit } 3 \\
\text { Pre }\end{array}$} & \multirow{2}{*}{$\begin{array}{l}\text { Visit } 4 \\
\text { Pre }\end{array}$} & \multirow{2}{*}{$\begin{array}{l}\text { Visit } 5 \\
\text { Pre }\end{array}$} \\
\hline & Baseline & Post (1 h) & & & \\
\hline \multicolumn{6}{|l|}{ Erythema } \\
\hline 0 : None & $0(0.0)$ & $2(10.5)$ & $0(0.0)$ & $4(21.1)$ & $0(0.0)$ \\
\hline 1: Trace & $4(21.1)$ & $5(26.3)$ & $11(57.9)$ & $14(73.7)$ & $11(57.9)$ \\
\hline 2: Mild & $15(78.9)$ & $12(63.2)$ & $8(42.1)$ & $1(5.3)$ & $8(42.1)$ \\
\hline $\begin{array}{l}\text { 3: Moder- } \\
\text { ate }\end{array}$ & $0(0.0)$ & $0(0.0)$ & $0(0.0)$ & $0(0.0)$ & $0(0.0)$ \\
\hline 4: Severe & $0(0.0)$ & $0(0.0)$ & $0(0.0)$ & $0(0.0)$ & $0(0.0)$ \\
\hline \multicolumn{6}{|l|}{ Edema } \\
\hline 0 : None & $0(0.0)$ & $0(0.0)$ & $2(10.5)$ & $10(52.6)$ & $16(84.2)$ \\
\hline 1: Trace & $10(52.6)$ & $12(63.2)$ & $16(84.2)$ & $9(47.4)$ & $3(15.8)$ \\
\hline 2: Mild & $9(47.4)$ & 7 (36.8) & $1(5.3)$ & $1(5.3)$ & $0(0.0)$ \\
\hline $\begin{array}{l}\text { 3: Moder- } \\
\text { ate }\end{array}$ & $0(0.0)$ & $0(0.0)$ & $0(0.0)$ & $0(0.0)$ & $0(0.0)$ \\
\hline 4: Severe & $0(0.0)$ & $0(0.0)$ & $0(0.0)$ & $0(0.0)$ & $0(0.0)$ \\
\hline \multicolumn{6}{|l|}{ Scaling } \\
\hline 0: None & $0(0.0)$ & $2(10.5)$ & $6(31.6)$ & $8(42.1)$ & $11(57.9)$ \\
\hline 1: Trace & $11(57.9)$ & $9(47.4)$ & $10(52.6)$ & $11(57.9)$ & $8(42.1)$ \\
\hline 2: Mild & 7 (36.8) & $8(42.1)$ & $3(15.8)$ & $0(0.0)$ & $0(0.0)$ \\
\hline $\begin{array}{l}\text { 3: Moder- } \\
\text { ate }\end{array}$ & $1(5.3)$ & $0(0.0)$ & $0(0.0)$ & $0(0.0)$ & $0(0.0)$ \\
\hline 4: Severe & $0(0.0)$ & $0(0.0)$ & $0(0.0)$ & $0(0.0)$ & $0(0.0)$ \\
\hline \multicolumn{6}{|c|}{ Stinging/burning } \\
\hline 0 : None & $1(5.3)$ & $2(10.5)$ & $8(42.1)$ & $13(68.4)$ & 15 (78.9) \\
\hline 1: Mild & $16(84.2)$ & $15(78.9)$ & $11(57.9)$ & $6(31.6)$ & $4(21.1)$ \\
\hline $\begin{array}{l}\text { 2: Moder- } \\
\text { ate }\end{array}$ & $2(10.5)$ & $2(10.5)$ & $0(0.0)$ & $0(0.0)$ & $0(0.0)$ \\
\hline 3: Severe & $0(0.0)$ & $0(0.0)$ & $0(0.0)$ & $0(0.0)$ & $0(0.0)$ \\
\hline \multicolumn{6}{|l|}{ Pruritus } \\
\hline 0: None & $8(42.1)$ & $10(52.6)$ & $6(31.6)$ & 9 (47.4) & $10(52.6)$ \\
\hline 1: Minimal & $11(57.9)$ & 9 (47.4) & $13(68.4)$ & $10(52.6)$ & $9(47.4)$ \\
\hline $\begin{array}{l}\text { 2: Moder- } \\
\text { ate }\end{array}$ & $0(0.0)$ & $0(0.0)$ & $0(0.0)$ & $0(0.0)$ & $0(0.0)$ \\
\hline 3: Severe & $0(0.0)$ & $0(0.0)$ & $0(0.0)$ & $0(0.0)$ & $0(0.0)$ \\
\hline
\end{tabular}

Data are expressed as $n(\%)$

Number of subjects $=19$

$L S R$ local skin reaction

\subsection{Detection of Clindamycin-Resistant C. acnes Strains in Acne Samples Collected from Patients}

C. acnes colonies were isolated from the samples collected from subjects and grown on BHI agar plates. Using the PCR method, the presence of the A2058G mutation on the $23 \mathrm{~S}$ rRNA gene, and ermX gene detection in DNA extracted from the cells of $C$. acnes colonies, were performed. The MboII restriction enzyme specifically recognises and cleaves DNA when there is a A2058G mutation in the bacterial target. The presence of two specific bands on agarose gel electrophoresis following restriction conferred the presence of the aforesaid mutation, which is responsible for clindamycin resistance. Similarly, presence of the ermX gene in the $C$. acnes DNA, which is responsible for clindamycin resistance in the strain, was checked using ermX-specific primers (data not shown).

Analysis of acne swab samples collected from 80 subjects on Visit 1 (during initial screening) revealed the presence of clindamycin-resistant $C$. acnes strains in swab samples of 19 subjects. Of these, strains with the A2058G mutation in the 50S ribosomal subunit were identified in swab samples of 10 subjects, samples from 7 subjects had C. acnes isolates that were ermX gene-positive, and swab samples from two subjects exhibited $C$. acnes strains positive for both the above-mentioned features (Fig. 3a). At Visit 3 (week 4), acne samples from 10 of 19 subjects enrolled displayed the presence of clindamycin-resistant $C$. acnes strains; this number further decreased to 8 on Visit 5 (week 12) after topical application of VB-1953 gel.

Using RT-PCR, we estimated the number of clindamycin-resistant $C$. acnes strains in the acne samples. Figure $3 \mathrm{~b}$ shows that the resistant $C$. acnes gradually decreased from $\log 4.9 \mathrm{CFU} / \mathrm{mL}$ on Visit 1 to $\log 3.6 \mathrm{CFU} / \mathrm{mL}$ on Visit 5, indicating a decrease in total resistant $C$. acnes by $94.3 \pm 1.0 \%(p<0.05)$ after 4 weeks of topical application of VB-1953.

\section{Discussion}

With the emergence of resistance to the currently approved antibiotics in clinical isolates of $C$. acnes, there is an urgent requirement to look beyond the existing arsenal of anti-acne agents $[18,40]$. While antimicrobials such as benzoyl peroxides are increasingly being used as an alternate choice [41, 42], clinical evidences show that such antimicrobials are maximally effective only when combined with antibiotics [26, 27]. Therefore, there is an unmet need for nextgeneration antibiotics that can retard the development of resistance, in addition to being effective against resistant strains of $C$. acnes. Antibiotics that are bactericidal in nature and can exert enhanced efficacy against $C$. acnes of varied resistance profiles, while mounting an anti-inflammatory effect to manage the host inflammation, could emerge as the next-generation treatment in the management of acne [18]. Such an active antibiotic when delivered at optimum concentrations at the desired site can emerge as a powerful treatment of acne.

Fluoroquinolones are known for their anti-inflammatory properties, apart from their strong antibacterial action [43]. Several evidences (both in vivo and in vitro) suggest the involvement of fluoroquinolones in the inhibition of LPS-induced tumour necrosis factor (TNF)- $\alpha$ and several 
Fig. 2 Efficacy of the application of VB-1953 topical gel (2\%) for 12 weeks twice daily for acne patients with moderate to severe acne. Effect of the application of VB-1953 topical gel $(2 \%)$ on acne a inflammatory and $\mathbf{b}$ noninflammatory lesion counts. The mean and SD are shown $(n=19)$. The mean absolute inflammatory lesion score decreased from $34.4 \pm 6.4$ at baseline to $16.7 \pm 9.0$ at week 12 ( $p<0.0001$; two-tailed $t$ test), whereas the noninflammatory lesion score decreased from $39.4 \pm 7.8$ at baseline to $19.5 \pm 10.0$ at week 12 $(p<0.0001$; two-tailed $t$ test). c Percentage reduction of the inflammatory and noninflammatory count across different weeks (baseline and weeks 4 , 8 and 12) during application of the VB-1953 topical gel (2\%) formulation. d IGA scores (\%) at baseline and week 12 . The IGA score for severity of acne has been graded as 0: clear; 1 : almost clear; 2: mild; 3: moderate; and 4: severe. $S D$ standard deviation, IGA Investigator's Global Assessment
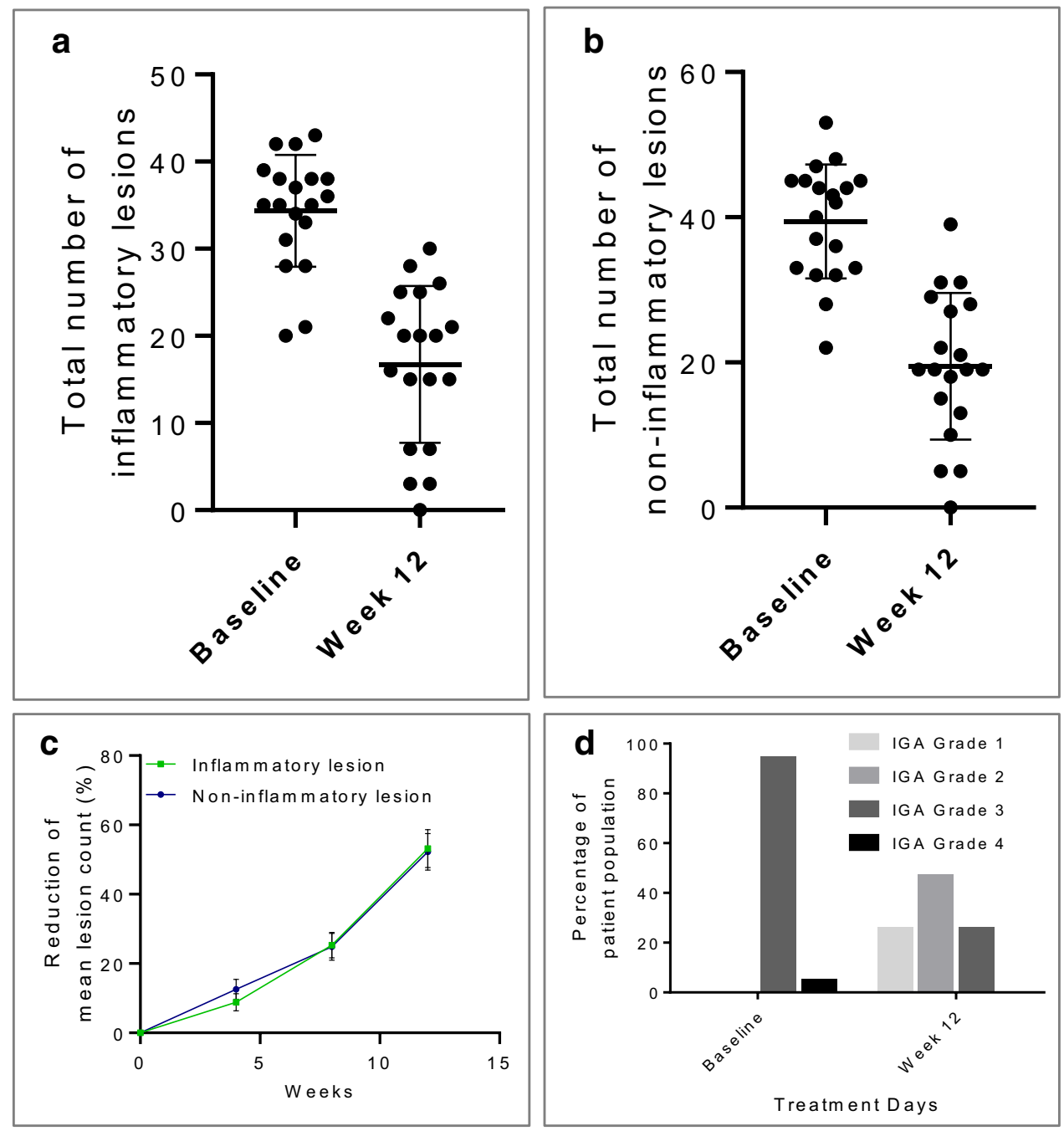

Fig. 3 Effect of the topical application of VB-1953 (2\%) gel on clindamycin-resistant strains associated with acne lesions. a Number of subjects harbouring resistant $C$. acnes at different weeks: initial screening date (Visit 1), week 4 after enrolment (Visit 3), and week 12 after enrolment (Visit 5). b Clindamycin-resistant $C$. acnes bacterial load (log CFU/mL) decreased at the initial screening date (Visit 1), week 4 after enrolment (Visit 3), and week 12 after enrolment (Visit 5). C. acnes Cutibacterium acnes, $C F U$ colony-forming unit

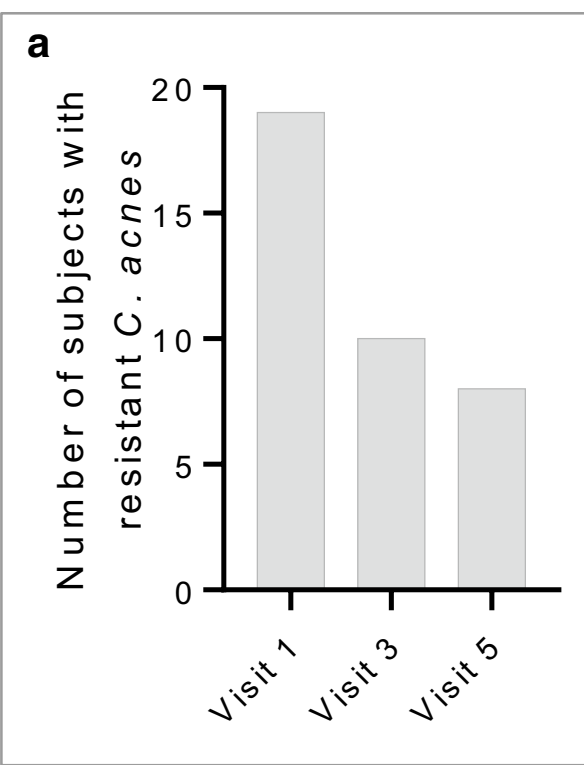


interleukins, such as IL-1 and IL-6 production [44, 45]. One of the critical components in acne is host-mediated inflammation. $C$. acnes is known to induce inflammatory cytokine expression, including TNF $\alpha$, IL-6, IL-8 and IL-12, in macrophages via activation of TLR-2 [8]. The role of IL-6 in $C$. acnes-induced cell lines, such as macrophages, keratinocytes and sebocytes, has been shown in multiple reports $[8,46$, 47]. Alestas et al. found enhanced IL-6 expression in acneinvolved skin [48]. The IL-1 family of cytokines has been found to be associated with tissue damage in acne patients [49]. VB-1953 was found to reduce dead C. acnes-induced inflammation in both in vitro and in vivo models. This action is independent of its bactericidal action. In vitro, VB-1953 significantly reduced IL-6 levels in the human monocyte cell line THP-1 [31]. Furthermore, VB-1953 showed a dosedependent reduction in messenger RNA (mRNA) expression of IL- $1 \alpha$, IL- $1 \beta$, IL-6, IL- 8 and IL-12 in THP- 1 cells (unpublished data). VB-1953 formulation was also found to exert anti-inflammatory effects in a dead $C$. acnes-induced rat paw edema model (unpublished data).

In this study, we selected a bactericidal antibiotic, VB-1953, a fourth-generation fluoroquinolone that is potent across $C$. acnes having different resistance phylotypes [18]. A topical formulation of VB-1953 showed a higher reduction in inflammatory lesion counts when applied once or twice daily compared with a pooled vehicle treatment group in a proof-of-concept clinical trial [31]. In this study, we report that 2\% VB-1953 was safe and well tolerated in moderate to severe acne patients with clindamycin-resistant $C$. acnes. Interestingly, there had been a significant decrease in both inflammatory and noninflammatory acne lesions within 12 weeks of application of the formulation, with a decrease in patients harbouring clindamycin-resistant $C$. acnes strains of $57.8 \%$ from baseline.

Of the 80 clindamycin nonresponder acne patients, 19 subjects were enrolled in the study after initial laboratory screening for the presence of clindamycin-resistant $C$. acnes on their acne lesion samples. This was one of the critical study criteria to evaluate the efficacy of the VB-1953 formulation in patients with clindamycin-resistant strains of C. acnes. Our data showed only $24 \%$ of the total subjects screened were associated with clindamycin-resistant $C$. acnes. It is possible that the remaining clindamycin nonresponder patients had additional etiologies that conferred the nonresponsive status; however, we only focused on those patients who met the dual criteria of clindamycin treatment failure together with harbouring clindamycin-resistant strains of $C$. acnes. It is possible that the percentage of this subset reflected the distribution in the population, verisimilar to the one in three patients reported in the US [17]. Additionally, the low prevalence could also be due to the method of sample collection, insufficient sampling area, surface sampling, or failure of sufficient growth of $C$. acnes colonies. However, we believe that this is a large subset of the population that can benefit from an effective drug rather than being treated with an antibiotic that they are nonresponsive to.

Lincosamides (clindamycin) are known to inhibit bacterial protein synthesis by interacting $23 \mathrm{~S}$ rRNA residues [50] in a similar way to macrolides. A2058 (E. coli numbering) residue is one of the pivotal nucleotides for lincosamide binding at the bacterial target [51]. Point mutation at A2058G in 23S rRNA is reported to confer high clindamycin resistance [52]. Similarly, many reports indicate instances of ermX gene-mediated clindamycin and erythromycin resistance in C. acnes [24]. In our case, 53\% of the 19 recruited subjects showed presence of clindamycin-resistant $C$. acnes strains with the A2058G mutation, $37 \%$ of cases were ermX gene-positive, and the remaining $10 \%$ showed presence of both the A2058G mutation and the ermX gene on their skin. Topical application of VB-1953 for 12 weeks on all 19 patients with clindamycin-resistant strains in their skin decreased this number from 19 to 8 . A decrease in resistant C. acnes count was also confirmed by RT-PCR. Over the 12-week treatment course, it was observed that the average colony count of resistant bacteria in the patient population was reduced by approximately $94 \%(p<0.05)$. VB- 1953 efficacy in ameliorating inflammatory and noninflammatory lesions $(p<0.001)$ in patients with acne lesions tested positive for clindamycin-resistant $C$. acnes offers an exciting clinical option for this subset of patients. It should be noted that these patients had not responded to clindamycin. Mechanistically, this could be attributed to the bactericidal action on a different target in C. acnes. Additionally, VB-1953 also exerts an immunomodulatory effect via decreasing proinflammatory cytokine-mediated inflammation (unpublished data). Taken together with the safety profile of VB-1953, our data strongly suggest that VB-1953 can emerge as the next-generation treatment for moderate to severe inflammatory acne, especially in the setting of clindamycin-resistant etiology. However, one of the major limitations of this study is that it was an open-label trial with a small sample size. A bigger, double-blinded study is warranted for conformation of the data with more than one clinical observer. Moreover, photographs of treated patients, if taken with proper consent, would have been helpful to support the efficacy of the treatment.

\section{Conclusion}

VB-1953 showcased good efficacy in treating both inflammatory and noninflammatory acne lesions without serious adverse effects. While larger studies are necessary to confirm these findings, our present data suggest VB-1953 as a future 
therapy against acne, even with an underlying resistant $C$. acnes etiology.

Author Contributions RB conducted the study and was involved in study conception and design. All the microbiology and molecular biology studies were designed and executed by Suresh Sadhasivam, Swati Gupta, Swamini Saini and Shamik Ghosh. All authors contributed to data analysis and interpretation of the data. RB participated in statistical analysis. RKSB, Suresh Sadhasivam, MS, Shamik Ghosh and SJ drafted the manuscript. All authors critically revised the manuscript and approved the final version.

\section{Compliance with Ethical Standards}

Funding Vyome Therapeutics provided the test articles free of charge for conducting the study, on the investigator's request.

Conflict of interest Rohit Batra is the Director of Dermaworld Skin and Hair Clinic and conducted the study. Suresh Sadhasivam, Swamini Saini, Swati Gupta, Rahul Kumar Singh Bisen, Mau Sinha, Shamik Ghosh and Shilpi Jain are employees of Vyome Therapeutics Limited. Shamik Ghosh holds equity in Vyome Therapeutics Limited.

Ethical approval This study was approved by the Independent Ethics Committee (GSER, Delhi, India) and was conducted as per Schedule Y (amended version, 2005) of the CDSCO, Ministry of Health and Family Welfare, Government of India; 'Ethical Guidelines for Biomedical Research on Human Participants' (2006); ICMR; ICH E6-(R1) 'Guideline for Good Clinical Practice'; and the Declaration of Helsinki (2013). Study-related procedures were initiated only after obtaining written informed consent from all subjects.

Open Access This article is licensed under a Creative Commons Attribution-NonCommercial 4.0 International License, which permits any non-commercial use, sharing, adaptation, distribution and reproduction in any medium or format, as long as you give appropriate credit to the original author(s) and the source, provide a link to the Creative Commons licence, and indicate if changes were made. The images or other third party material in this article are included in the article's Creative Commons licence, unless indicated otherwise in a credit line to the material. If material is not included in the article's Creative Commons licence and your intended use is not permitted by statutory regulation or exceeds the permitted use, you will need to obtain permission directly from the copyright holder.To view a copy of this licence, visit http://creativecommons.org/licenses/by-nc/4.0/.

\section{References}

1. Bhate K, Williams HC. Epidemiology of acne vulgaris. Br J Dermatol. 2013;168(3):474-85.

2. Tan JK, Bhate K. A global perspective on the epidemiology of acne. Br J Dermatol. 2015;172(Suppl 1):3-12.

3. Moradi Tuchayi S, Makrantonaki E, Ganceviciene R, Dessinioti C, Feldman SR, Zouboulis CC. Acne vulgaris. Nat Rev Dis Primers. 2015;1:15029.

4. Burke BM, Cunliffe WJ. The assessment of acne vulgaris: the Leeds technique. Br J Dermatol. 1984;111(1):83-92.

5. Webster GF. Acne vulgaris. BMJ. 2002;325(7362):475-9.
6. Cunliffe WJ, Clayden AD, Gould D, Simpson NB. Acne vulgaris: its aetiology and treatment. A review. Clin Exp Dermatol. 1981;6:461-9.

7. Vowels BR, Yang S, Leyden JJ. Induction of proinflammatory cytokines by a soluble factor of Propionibacterium acnes: implications for chronic inflammatory acne. Infect Immun. 1995;63(8):3158-65.

8. Kim J, Ochoa MT, Krutzik SR, Takeuchi O, Uematsu S, Legaspi $\mathrm{AJ}$, et al. Activation of toll-like receptor 2 in acne triggers inflammatory cytokine responses. J Immunol. 2002;169:1535-41.

9. Graham GM, Farrar MD, Cruse-Sawyer JE, Holland KT, Ingham E. Proinflammatory cytokine production by human keratinocytes stimulated with Propionibacterium acnes and P. acnes GroEL. Br J Dermatol. 2004;150(3):421-8.

10. Grange PA, Raingeaud J, Calvez V, Dupin N. Nicotinamide inhibits Propionibacterium acnes-induced IL-8 production in keratinocytes through the NF-kappaB and MAPK pathways. J Dermatol Sci. 2009;56(2):106-12.

11. Lee SE, Kim JM, Jeong SK, Jeon JE, Yoon HJ, Jeong MK, et al. Protease-activated receptor- 2 mediates the expression of inflammatory cytokines, antimicrobial peptides, and matrix metalloproteinases in keratinocytes in response to Propionibacterium acnes. Arch Dermatol Res. 2010;302(10):745-56.

12. Li ZJ, Choi DK, Sohn KC, Seo MS, Lee HE, Lee Y, et al. Propionibacterium acnes activates the NLRP3 inflammasome in human sebocytes. J Invest Dermatol. 2014;134(11):2747-56.

13. Tsai HH, Lee WR, Wang PH, Cheng KT, Chen YC, Shen SC. Propionibacterium acnes-induced iNOS and COX-2 protein expression via ROS-dependent NF- $\mathrm{KB}$ and AP-1 activation in macrophages. J Dermatol Sci. 2013;69(2):122-31.

14. Leyden JJ. Therapy for acne vulgaris. N Engl J Med. 1997;336(16):1156-62.

15. Aslam I, Fleischer A, Feldman S. Emerging drugs for the treatment of acne. Expert Opin Emerg Drugs. 2015;20(1):91-101.

16. Leyden JJ, McGinley KJ, Cavalieri S, Webster GF, Mills OH, Kligman AM. Propionibacterium acnes resistance to antibiotics in acne patients. J Am Acad Dermatol. 1983;8:41-5.

17. Ross JI, Snelling AM, Eady EA, Cove JH, Cunliffe WJ, Leyden $\mathrm{JJ}$, et al. Phenotypic and genotypic characterization of antibioticresistant Propionibacterium acnes isolated from acne patients attending dermatology clinics in Europe, the U.S.A., Japan and Australia. Br J Dermatol. 2001;144(2):339-46.

18. Sinha M, Sadhasivam S, Bhattacharyya A, Jain S, Ghosh S, Arndt KA, et al. Antibiotic-resistant acne: getting under the skin. Semin Cutan Med Surg. 2016;35(2):62-7.

19. Coates P, Vyakrnam S, Eady EA, Jones CE, Cove JH, Cunliffe WJ. Prevalence of antibiotic-resistant propionibacteria on the skin of acne patients: 10-year surveillance data and snapshot distribution study. Br J Dermatol. 2002;146(5):840-8.

20. Giannopoulos L, Papaparaskevas J, Refene E, Daikos G, Stavrianeas N, Tsakris A. MLST typing of antimicrobial-resistant Propionibacterium acnes isolates from patients with moderate to severe acne vulgaris. Anaerobe. 2015;31:50-4.

21. Sardana K, Gupta T, Garg VK, Ghunawat S. Antibiotic resistance to Propionobacterium acnes: worldwide scenario, diagnosis and management. Expert Rev Anti Infect Ther. 2015;13:883-96.

22. Ross JI, Eady EA, Cove JH, Jones CE, Ratyal AH, Miller YW, et al. Clinical resistance to erythromycin and clindamycin in cutaneous propionibacteria isolated from acne patients is associated with mutations in $23 \mathrm{~S}$ rRNA. Antimicrob Agents Chemother. 1997;41(5):1162-5.

23. McDowell A, Barnard E, Nagy I, Gao A, Tomida S, Li H, et al An expanded multilocus sequence typing scheme for Propionibacterium acnes: investigation of 'pathogenic', 'commensal' and antibiotic resistant strains. PLoS One. 2012;7(7):e41480. 
24. Ross JI, Eady EA, Carnigie E, Cove JH. Detection of transposon Tn 5432-mediated macrolide-lincosamide-streptogramin B (MLSB) resistance in cutaneous propionibacteria from six European cities. J Antimicrob Chemother. 2002;49(1):165-8.

25. Ross JI, Eady EA, Cove JH, Cunliffe WJ. 16S rRNA mutation associated with tetracycline resistance in a gram-positive bacterium. Antimicrob Agents Chemother. 1998;42(7):1702-5.

26. Nast A, Dreno B, Bettoli V, Degitz K, Erdmann R, Finlay AY, et al. European evidence based (S3) guidelines for the treatment of acne. J Eur Acad Dermatol Venereol. 2012;26(Suppl 1):1-29.

27. Thiboutot D, Gollnick H, Bettoli V, Dréno B, Kang S, Leyden JJ, et al. New insights into the management of acne: an update from the Global Alliance to Improve Outcomes in Acne group. J Am Acad Dermatol. 2009;60(5 Suppl):S1-50.

28. Hooper DC. Mechanisms of action of antimicrobials: focus on fluoroquinolones. Clin Infect Dis. 2001;32(Suppl 1):S9-15.

29. Kurokawa I, Akamatsu H, Nishijima S, Asada Y, Kawabata S. Clinical and bacteriologic evaluation of OPC-7251 in patients with acne: a double-blind group comparison study versus cream base. J Am Acad Dermatol. 1991;25(4):674-81.

30. Plewig G, Holland KT, Nenoff P. Clinical and bacteriological evaluation of nadifloxacin $1 \%$ cream in patients with acne vulgaris: a double-blind, phase III comparison study versus erythromycin 2\% cream. Eur J Dermatol. 2006;16(1):48-55.

31. Jain S, Secci A. A proof of concept clinical study to test the efficacy and safety of VB-1953 in moderate to severe acne subjects. J Invest Dermatol. 2019;139(5):S107.

32. Dréno B, Bettoli V, Ochsendorf F, Layton AM, Perez M, Dakovic $\mathrm{R}$, et al. Efficacy and safety of clindamycin phosphate $1.2 \%$ tretinoin $0.025 \%$ formulation for the treatment of acne vulgaris: pooled analysis of data from three randomised, double-blind, parallel-group, phase III studies. Eur J Dermatol. 2014;24(2):201-9.

33. Zaenglein AL, Pathy AL, Schlosser BJ, Alikhan A, Baldwin HE, Berson DS, et al. Guidelines of care for the management of acne vulgaris. J Am Acad Dermatol. 2016;74(5):945-973.e33.

34. Jain S, Yadav V, Bhatia N. Clinical pharmacokinetics, safety and exploratory efficacy study of a topical bactericidal VB-1953: analysis of single and multiple doses in a phase I trial in acne vulgaris subjects. Clin Drug Investig. 2020;40(3):259-68.

35. Leyden JJ, Preston N, Osborn C, Gottschalk RW. In-vivo effectiveness of adapalene $0.1 \%$ /benzoyl peroxide $2.5 \%$ gel on antibioticsensitive and resistant Propionibacterium acnes. J Clin Aesthet Dermatol. 2011;4(5):22.

36. Bojar RA, Eady EA, Jones CE, Cunliffe WJ, Holland KT. Inhibition of erythromycin-resistant propionibacteria on the skin of acne patients by topical erythromycin with and without zinc. Br J Dermatol. 1994;130(3):329-36.

37. Muller EE, Paz-Bailey G, Lewis DA. Macrolide resistance testing and molecular subtyping of Treponema pallidum strains from southern Africa. Sex Transm Infect. 2012;88(6):470-4.

38. Afonina IA, Reed MW, Lusby E, Shishkina IG, Belousov YS. Minor groove binder-conjugated DNA probes for quantitative DNA detection by hybridization-triggered fluorescence. Biotechniques. 2002;32(4):940-9.
39. Hao H, Liu J, Kuang X, Dai M, Cheng G, Wang X, et al. Identification of Campylobacter jejuni and determination of point mutations associated with macrolide resistance using a multiplex TaqMan MGB real-time PCR. J Appl Microbiol. 2015;118(6):1418-25.

40. Simonart T, Dramaix M. Treatment of acne with topical antibiotics: lessons from clinical studies. Br J Dermatol. 2005; 153:395-403.

41. Mills OH Jr, Kligman AM, Pochi P, Comite H. Comparing 2.5\%, $5 \%$, and $10 \%$ benzoyl peroxide on inflammatory acne vulgaris. Int J Dermatol. 1986;25:664-7.

42. Strauss JS, Krowchuk DP, Leyden JJ, Lucky AW, Shalita AR, Siegfried EC, et al. Guidelines of care for acne vulgaris management. J Am Acad Dermatol. 2007;56(4):651-63.

43. Dalhoff A. Immunomodulatory activities of fluoroquinolones. Infection. 2005;33(Suppl 2):55-70.

44. Choi JH, Song MJ, Kim SH, Choi SM, Lee DG, Yoo JH, et al. Effect of moxifloxacin on production of proinflammatory cytokines from human peripheral blood mononuclear cells. Antimicrob Agents Chemother. 2003;47(12):3704-7.

45. Ogino H, Fujii M, Ono M, Maezawa K, Hori S, Kizu J. In vivo and in vitro effects of fluoroquinolones on lipopolysaccharideinduced pro-inflammatory cytokine production. J Infect Chemother. 2009;15(3): 168-73.

46. Huang YC, Yang CH, Li TT, Zouboulis CC, Hsu HC. Cell-free extracts of Propionibacterium acnes stimulate cytokine production through activation of $\mathrm{p} 38$ MAPK and Toll-like receptor in SZ95 sebocytes. Life Sci. 2015;139:123-31.

47. Lee WJ, Jung HD, Chi SG, Kim BS, Lee SJ, Kim DW, et al. Effect of dihydrotestosterone on the upregulation of inflammatory cytokines in cultured sebocytes. Arch Dermatol Res. 2010;302:429-33.

48. Alestas T, Ganceviciene R, Fimmel S, Müller-Decker K, Zouboulis CC. Enzymes involved in the biosynthesis of leukotriene B4 and prostaglandin E2 are active in sebaceous glands. J Mol Med (Berl). 2006;84:75-87.

49. Ingham E, Eady EA, Goodwin CE, Cove JH, Cunliffe WJ. Proinflammatory levels of interleukin-1 alpha-like bioactivity are present in the majority of open comedones in acne vulgaris. J Invest Dermatol. 1992;98:895-901.

50. Schlünzen F, Zarivach R, Harms J, Bashan A, Tocilj A, Albrecht $\mathrm{R}$, et al. Structural basis for the interaction of antibiotics with the peptidyl transferase centre in eubacteria. Nature. 2001;413(6858):814-21.

51. Douthwaite S. Interaction of the antibiotics clindamycin and lincomycin with Escherichia coli $23 \mathrm{~S}$ ribosomal-RNA. Nucleic Acids Res. 1992;20(18):4717-20.

52. Sadhasivam S, Sinha M, Saini S, Kaur SP, Gupta T, Sengupta S, et al. Heterogeneity and antibiotic resistance in Propionibacterium acnes isolates and its therapeutic implications: blurring the lines between commensal and pathogenic phylotypes. Dermatol Ther. 2016;29(6):451-4. 